\title{
Localized moving breathers in a 2-D hexagonal lattice
}

\author{
J. L. Marín*, J. C. Eilbeck ${ }^{\dagger}$, and F. M. Russell \\ Department of Mathematics, Heriot-Watt University, \\ Edinburgh EH14 4AS, U.K.
}

(November 13, 2018)

\begin{abstract}
We show for the first time that highly localized in-plane breathers can propagate in specific directions with minimal lateral spreading in a model 2-D hexagonal non-linear lattice. The lattice is subject to an on-site potential in addition to longitudinal nonlinear inter-particle interactions. This study investigates the prediction that stable breather-like solitons could be formed as a result of energetic scattering events in a given layered crystal and would propagate in atomic-chain directions in certain atomic planes. This prediction arose from a long-term study of previously unexplained dark lines in natural crystals of muscovite mica.
\end{abstract}

63.20.Pw, 63.20.Ry, 03.40.Kf.

The response of a nonlinear 2D atomic lattice when embedded in a surrounding 3D lattice is presently of much interest in connection with transport phenomena in layered crystals. Two examples of such crystals are the copper-oxide based high temperature superconductors and the potassium based silicate muscovite mica. Fortunately the optical transparency of the latter allows the study of energetic events at the atomic level. This is possible in mica because of tracks made visible by impurities of transient defects, through triggered solid-state phase transitions. A study of these tracks arising from nuclear scattering events led to the suggestion that energy could be transported over large distances through the crystal by some sort of energetic intrinsic localized mode (ILM) on the lattice [1]. The interesting and novel aspect of this prediction was that these ILMs would travel along the crystal axes and remain localized in both longitudinal and transverse directions with little or no lateral spreading. This is despite the fact that the $2 \mathrm{D}$ lattice has full hexagonal symmetry. This one-dimensional behavior in a two-dimensional lattice was called quasi-onedimensional (QOD) and the resulting ILMs were called "quodons" [2].

The purpose of this present letter is to demonstrate numerical simulations of a mica-like model which support the QOD conjecture. Our intention is not to provide a detailed model of the mica system at this stage, but merely to present a simplified model which maintains much of the overall qualitative features of the mica system and yet can be easily studied. The model is general enough to suggest the possibility of QOD effects in other hexagonal crystal structures, and even other geometries such as square lattices.

Muscovite mica has a layered structure. A conspicuous feature of this structure is the mono-atomic planes of

*marin@wanda.unizar.es

†chris@ma.hw.ac.uk 
potassium atoms with large interatomic spacing, tightly sandwiched between planes containing oxygen atoms, the oxygen atoms bound in compact silicate layers which impose a hexagonal structure. The silicon atoms in these silicate layers also lie in planes but are further away from the potassium planes. These features are indicated in Fig. 1, which shows the symmetry about the potassium plane. We are interested here only in the dynamics of the potassium atoms when moving in their 2D hexagonal plane as a result of in-plane impulses. The motion of the potassium atoms about their equilibrium positions is influenced strongly by the adjacent planes of oxygen atoms, which form a nearly rigid lattice and therefore give rise to strong on-site potentials.

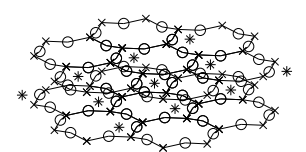

(a)

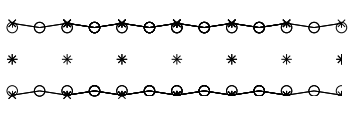

(b)

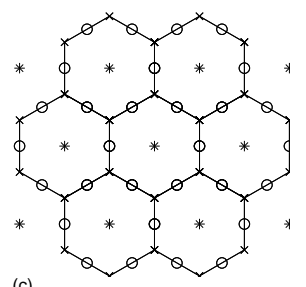

FIG. 1. The layered structure of muscovite mica. Oxygen atoms are marked by $\circ$, the silicon atoms by $\times$, and the potassium atoms by $*$.

We assume the potassium atoms are subject to a nonlinear coupling with the other potassium atoms, and to a nonlinear force due to the fixed oxygen atoms in the planes above and below. The (classical) Hamiltonian of the system is:

$H=\sum_{i, j}\left[\frac{1}{2} \dot{\vec{u}}_{i, j}^{2}+\alpha V\left(\vec{u}_{i, j}\right)+\frac{1}{2} \lambda \sum_{i^{\prime}, j^{\prime}} W\left(\vec{u}_{i, j}, \vec{u}_{i^{\prime}, j^{\prime}}\right)\right]$

where the indexes $(i, j)$ represent the coordinates of the atomic (equilibrium) positions in terms of a lattice basis of vectors $(\vec{a}, \vec{b})$. We will denote the lattice spacing by $a=$ $|\vec{a}|=|\vec{b}|$. The vectors $\vec{u}$ represent relative displacements from these positions. The primed quantities represent the interacting neighbors for each site, and the $\frac{1}{2}$ factor is there to avoid double counting interactions. In our case, we have restricted our simulations to nearest neighbor interactions. We use radial potentials for both the onsite and the K-K potentials.

For the interatomic K-K potential we use

$$
W\left(\vec{u}, \vec{u}^{\prime}\right)=W\left(\left|\vec{u}-\vec{u}^{\prime}-\vec{c}\right|\right)=W(|\vec{d}|)
$$


where $\vec{c}$ is the equilibrium relative position vector, that is, a lattice vector with $|\vec{c}|=a . \vec{d}=\vec{u}-\vec{u}^{\prime}-\vec{c}$ is the actual separation vector between the $(i, j)$ and the $\left(i^{\prime}, j^{\prime}\right)$ th sites. The functional form of $W$ is chosen to be a classical 6-12 Lennard-Jones potential

$$
W_{L J}(r)=1+\left(\frac{a}{r}\right)^{12}-2\left(\frac{a}{r}\right)^{6} ; \quad r \equiv|\vec{d}|
$$

where the normalization has been chosen in such a way that the equilibrium position is at $r=a$ (the lattice constant) and, for convenience, with energy minimum zero.

For the on-site potential, we mimic the effect of the 6 oxygen atoms in each of the two silicate planes above and below the K plane by a "virtual" oxygen atom situated in the same position as each $\mathrm{K}$ atom:

$$
V(\vec{u})=\sum_{k=1}^{6} V_{o}\left(\left|\vec{u}-\vec{c}_{k}\right|\right)
$$

where $V_{o}(x)$ is the potential due to each of the 6 fixed virtual oxygen atoms at lattice positions $\vec{c}_{k}, k=1, \ldots, 6$. Since the lattice modes we have explored are highly anharmonic and thus we expect large displacements of the potassium atoms, we have chosen a standard Morse potential for this interaction:

$$
V_{o}(s)=\frac{1}{2}(1-\exp (-s))^{2} ; \quad s \equiv\left|\vec{u}-\vec{c}_{k}\right|-a
$$

This form of interaction does not have a hard-core repulsion and would allow the the potassium atoms to "pass through" the virtual oxygens, which is a better approximation to the on-site effective potential we are trying to simulate. On the other hand one has to be careful with the relative scalings between potentials (3) and (2), otherwise one could dominate the other for large displacements. It can be checked that for $a \simeq 7$ we have an adequate balance between the two for the typical displacements involved in the lattice modes studied.

We show the result of a typical numerical run in Fig. 2. Simulations are shown on a $16 \times 16$ lattice, we have reproduced similar results on larger lattices. For the numerical integration we used efficient symplectic methods [3]. Initially, we explored different strategies to obtain moving nonlinear modes in this lattice. It turned out that the most successful and easy way to generate such solutions was hinted by the analogue phenomenon of intrinsic localization in 1D nonlinear lattices. Intrinsic localization of non-moving modes is well-known and its genericity has been rigorously established. Moving modes are not so well studied, but they appear generically in simulations of 1D nonlinear lattices, and in some special cases have been numerically calculated to high accuracy 沺. 

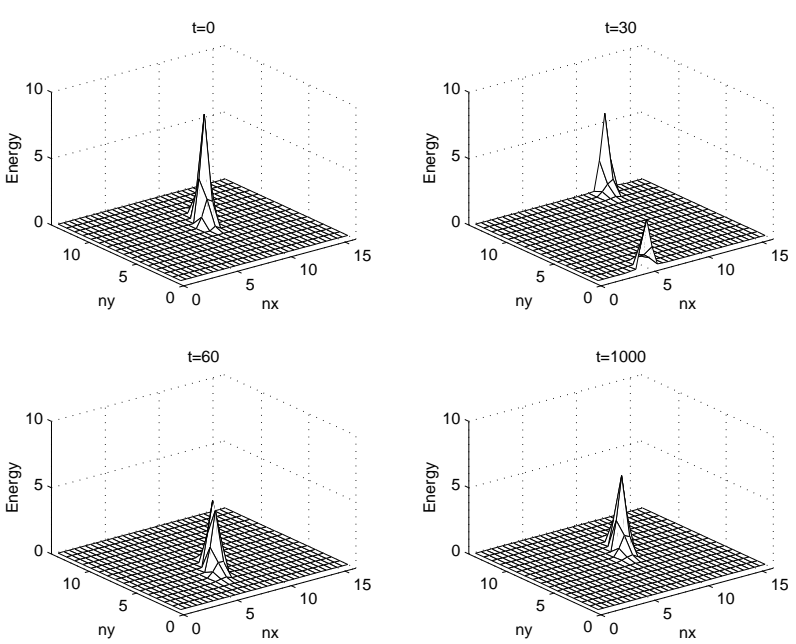

FIG. 2. Evolution of the energy density profile.

Based on experience with 1D lattices, we tried to generate quasi-one-dimensional moving modes by exciting a few consecutive atoms, with excitations parallel to their crystal axis. The simulation shown in Fig. 2 was obtained simply by exciting three atoms with a pattern of velocities $-2,4,1$, while the initial positions remained at their equilibrium points. The rest of the lattice atoms were at rest. With this choice of initial conditions we obtained a remarkably stable moving breather which propagates along a crystal axis with almost no lateral spreading.

Fig. 2(a) shows the initial distribution of energy, mainly concentrated on the three initially disturbed atoms (although some energy now resides in adjacent atoms due to the inter-particle forces). The peak moves due to the direction of the imparted momentum, along the crystal axis away from the position of the viewer. During the first few time units the peak also modifies its form, leaving behind some energy, until it has achieved a profile which apparently moves through the lattice with very little further distortion. Fig. 2(b) shows the result at $t=30$, when it is passing through the boundary at the far end of the rectangle and re-appearing on the near edge (due to the periodic boundary conditions) which clearly shows the direction of travel. Fig. 2(c) shows the pulse at $t=60$, after it has traversed the lattice once. Finally, Fig. 2(d) shows the pulse at a much later time of $t=1000$ when it has traversed the lattice 12 times, i.e. traveled 192 lattice sites. Further integration up to at least $t=7000$ (not shown) shows no appreciable degredation.

A close study of the solution shows that most of the energy is contained at any one time in three or four collinear atoms, with the surrounding atoms (including those one atomic spacing perpendicular to the direction of motion) sharing very small amounts of energy. This channeling of energy in a very localized way is an interesting phenomena, we are still studying the details of the mechanism, but it may be that some sort of discrete self-focusing 
effect is playing a part. Plots (not shown) of the displacements show the characteristic out-of phase motion of an optical breather 5]. In the first few tens of time units the pulse sheds some $10 \%$ of its local energy to the surrounding lattice, thereafter it adopts a more robust shape which loses energy at a much lower rate.

Further studies with a variety of initial conditions have shown that this is a robust phenomena for a range of parameter values. It has been observed that the initial velocity perturbations are allowed to deflect as much as $\pm 15^{\circ}$ and still produce the moving breather. However, there are threshold effects: if the initial disturbance is too weak then the breather will disperse in all directions, which demonstrates the essential importance of nonlinearity in the QOD behavior; on the other hand, if it is too strong then the breather will generate stronger radiation and will eventually become pinned by the lattice. It is even possible to generate a breather by giving just a single atom a nonzero velocity, but in this case the breather loses more energy to the surrounding atoms before forming itself into a stable moving structure. This remarkable behavior supports strongly the conjecture of quodons (moving breathers) being formed in mica by energetic cosmic rays, which would produce a large recoil in a single potassium atom. From our preliminary studies at least, it seems to be important that the strengths of the on-site and intersite potentials be roughly similar. More details of these effects, and of the effects of collision between two breathers, will be reported elsewhere.

A straightforward but lengthy calculation can be performed to calculate the wave speeds of small-amplitude waves in this model. From this we find that the velocity of the observed ILMs are sub-sonic, propagating at between $20 \%$ and $60 \%$ of the maximum group velocity of linear waves.

Although some work has been done on one-component breathers in a two-dimensional discrete sine-Gordon model [6], we believe that this is the first observation of mobile breathers in a two-component (i.e. displacement in both $x$ - and $y$-directions) 2D discrete lattice. More work is required to compare the model potentials used here with the molecular calculations of the full mica structure. It would be interesting also to see if similar results can be found in $2 \mathrm{D}$ cubic structures and in full $3 \mathrm{D}$ structures.

\section{Acknowledgments}

One of us (JLM) would like to thank the TRACS programme at the Edinburgh Parallel Computing Centre for support during a three month stay at Heriot-Watt University, during which time much of this work was done. We also thank J-C Desplat for his help with visualization software. We are grateful to E. Abraham, J. Blackburn, 
D. B. Duncan, B. Fleming and Y. Zolotaryuk for useful discussions.

[1] J. W. Steeds, F. M. Russell, and W. J. Vine, Optik 92, 149 (1993).

[2] F. M. Russell and D. R. Collins, Nuclear instruments \& methods in physics research B 105, 1 (1995).

[3] J. M. Sanz-Serna and M. P. Calvo, Numerical Hamiltonian Problems (Chapman and Hall, London, 1994).

[4] D. B. Duncan, J. C. Eilbeck, H. Feddersen, and J. A. D. Wattis, Physica D 68, 1 (1993).

[5] S. Flach, Phys. Rev. E 51, 3579 (1995), and references therein.

[6] J. M. Tamga, M. Remoissenet, and J. Pouget, Phys. Rev. Lett. 75, 357 (1995). 